Research article

\title{
Spatial mapping and enshrined capacity: Assessment of COVID-19 servicing Indian hospitals
}

\author{
N. Ravichandran*, S. Shivangi and B. Rakesh \\ Department of Healthcare and Pharmaceutical Management, Jamia Hamdard, Hamdard University, New Delhi-110062, \\ India
}

*Corresponding Author. E-mail: drnrav@gmail.com

\section{Article history}

Received : January 14, 2021

Accepted : January 29, 2021

Keywords

Coronavirus COVID-19 pandemic Frontline workers PPE kits

\begin{abstract}
In Public health crises like COVID-19, healthcare services alone won't essentially cause the wellliked changes within the health status and outcome. Human resources with needed competency for managing healthcare crisis could be a challenge, and can't be over accentuated. A systemic approach adopted to analyze knowledge, management, and delivery of COVID-19 services in the Indian context while the concentration curve alongside regression statistical techniques was used to examine the nature of competency and skill variations among the health-functionaries. Training processes on COVID-19 aren't streamlined and systematic. The method of organizing a training programme depends upon the need-based mostly. This mirrored within the variability of healthfunctionaries reported with adverse events, infected with the coronavirus. Several COVID-19 strategies focused on healthcare-functionaries and its associated front-liners to save lots of lives and alter the lifestyles of the population. The stigmatized COVID-19 disease brutally distanced the frontline health-workers and socially distanced the sufferers' delay in reporting, with heightened morbidity and mortality. Training is just on information rather than on competencies for action. The quality training and the level of community-based health intervention flaunted not to expect health functionaries to perform expectedly. Training-competency and skills-related inequality and inequity in health exist. There's a necessity to sources information equitably to empower the healthcare providers to deliver service effectively.
\end{abstract}

\section{INTRODUCTION}

Human resources are critical for the effective implementation of healthcare services delivered equitably to achieve sustainable development goals. The availability of the healthcare personnel with needed competency for managing healthcare crisis could be a challenge, and can't be over accentuated. In Public health crises like COVID19 , numbers alone won't essentially cause the well-liked changes within the health status and outcome. The reason's simple: we tend to, as human beings never learned a lesson from the past. Since WWII, the world has encountered a spread of endemic although human-beings might succeed some and succumbed to several. Mankind is proudly saying he's developed advanced medical tools and improved drug missionary to face challenges that occurred from time-to-time. However, the failure is - uncertainty in (medical) science and its unpredictable result orientation, because it never had preventive and promotive thoughts, rather centred on care and treatment. For instance, SARS jolted the world in 2003; after a decade, Ebola jerked in 2013 whereas COVID-19 agitated humanity in 2020 with no-time.

The repetitive influx of endemic proved inefficiency and ineffectiveness within the healthcare system, which demands 3-Cs - concern, commitment, and competencies alongside 5-As - available, accessible, affordable, approachable, and accountable. COVID-19 tried that the globalization forged in negative navigation in terms of accrued economic and doubled the disease burden. Little or no governance in informatics in conjunction with the evolved business competition with no or fewer ethics counterfeited COVID-19 pandemic. Several strategies are fabricated to arrest the invisible coronavirus, but paid the heavy toll, in terms of morbidity and mortality, without any benefits. Additionally, $50 \%$ of the productive population recorded with job loss; $32 \%$ of the population tumbled to the poverty line; $42 \%$ population begged to health poverty level and more than 460 million population go acute food shortage, each micro, and macroeconomy scrambled to a recession, a scary sketch to humanity and its livelihoods. This present involves 'design thinking in health-system and disease management'.

Managed care through integrated-medicine is also failed to display its competency although integrativemedicine has a competent to contribute to prophylaxis and protection of vulnerable organs involved in viral entry. Several COVID-19 strategies simply focused on healthcare-personnel and its associated front-liners to save lives and alter the lifestyles of the population (Misra, 2020). The stigmatized COVID-19 brutally distanced the frontline workers and socially distanced the sufferers' 
delay in reporting, with heightened morbidity and mortality. 'Nations without borders' revealed the gaps in COVID-19 management while didn't examine WWWW where we went wrong? This could be attributed to many factors: systemic issues, inequity, low healthcare financing, misinformation, and poor competencies in delivering services. This paper primarily addresses COVID-19 serving healthcare-functionaries and their training needs concerning KAD - Knowledge, Management, and Delivery in the Indian context. The present status of competency and skills raises many queries. Do healthcare functionaries lack the competence and skills to implement intervention correctly? Will the system have the potentiality to create the capability of those health-functionaries? Is that the standard training appropriate? Is there a preparedness embedded within the health system to develop the requisite skills in providing services effectively?

\section{RESEARCH APPROACH AND ITS ORIENTATION}

The study adopted a systemic approach to analyze KAD in the Indian context and understand factors and constraints in training for capacity-building and skills development for the services delivery. A multipronged procedure was used to collect, collate, and content of the data. A triage orientation was performed with a combination of asking questions, making observations, and reviewing the relevant records and reports. On-site recordings/observations were made to get first-hand knowledge of issues, and concerns from 621 healthfunctionaries who're engaged COVID-19 services during March-September 2020. A total of $25.9 \%$ of the respondents are medical-practitioners, 58\% nurses, $14.2 \%$ are paramedical and a few belong to caregivers. To assess the degree of effectiveness of the training and its inequalities, the concentration curve alongside regression statistical techniques were used to understand the nature of competency and skill variations among the health functionaries.

\section{TRAINING POLICY AND INFRASTRUCTURE}

Since the outbreak in Wuhan and misinformation regarding human-to-human transmission led the world to remain in sleeper-cell. This cost huge morbidity and human loss suggest an increase in healthcare and disease burden. Despite coronavirus unfold to over 180 countries affected with a 75-million population, no serious efforts are made to develop a comprehensive training policy. Lack of training policies to counter emerging endemic-COVID19 is due to low priority on human resource development and wrong perception regarding COVID-19 as flu-like. In India, many skill-development programs are initiated within the last 5-years on an ad-hoc basis whereas healthcare arising with and its associated human resources development have not yielded any expected results. For instance, aside from COVID-19 related healthcare services are suspended abruptly as healthcare professionals struggle to care and supply the appropriate treatment for patients with co-morbidities (78\%). This further put hospitals that are suspended its services are finding their inexistence or survival at the chaos. The coronavirus has humbled and humiliated humanity as we stand puzzled and stranded as personal protection equipment (PPE) and required standalone infrastructure aren't available. India isn't an exception to the present. India's dependency on PPE, medical instruments, diagnostic kits, and clinical knowhow led the population more anxious, bothered, concerned, and distressed-ABCD. Whereas Indians are put under strict lockdown alongside social distancing protocols, as India needed to import over $80 \%$ of its pharmacy/drugs raw materials although it's third-largest drug manufacturers in the world. It's understood that Indians shall not be panic however got to steer from the ill-equipped infrastructure. Meanwhile, Indian public-private-partnership went on to toss and hit. To strengthen its capacity, the government ordered the suspension of various health services to form extra space for COVID-19-patients and acquired other hospital beds with the existing paraphernalia and struck off private hospitals that didn't have isolated premises (MoHFW, 2020). This reflects the infrastructural constraints that desire attention to deal with uncertainty. Interaction with private-hospitals-association mirrored that there's not an organic link between public-private establishments for training, sharing of the knowledge and experience.

\section{TRAINING PROCESS}

A total of $37.5 \%$ male medical-practitioners involved in door-to-door screening; out of those involved, $31.3 \%$ and $43.5 \%$ stationed at secondary and tertiary setups than their feminine counterparts $(10.9 \%)$. In the nursing category, $41.9 \%$ and $12.5 \%$ male-nurses are based at secondary and tertiary hospitals compared to their feminine counterparts, $15.9 \%$, and $81 \%$ are so. Whereas $25 \%$ of male paramedical engaged in community door-todoor observance while $56.3 \%$ and $14.5 \%$ respectively are at secondary and tertiary hospitals' services compared to only $9.4 \%$ of females paramedical at tertiary setups.

Fig. 1 illustrates the majority shortage of information on how to use PPE whereas $15.3 \%$ of health-functionaries in secondary/tertiary hospitals out-rightly not having access to PPE. 54.3\% unconcealed that a major source of information regarding COVID-19 is internet sources whereas training plays a bare role (22.4\%). An insignificant percentage of medical-practitioners $(0.6 \%)$ and nurses $(1.2 \%)$ based on secondary/tertiary hospitals undergone training. A combination of self-learning through internet resources and training appears to be base for nurses $(21.6 \%)$ followed by paramedical staff $(18.3 \%)$ and medical-practitioners $(8.6 \%)$. Candidly, $23 \%$ of health-functionaries expressed that they receive information from all sources.

This study noted leadership isn't regular, no master trainers' cadre established, which they don't seem to be a worry. The training target is on information rather than on competencies for action. As a result, there is no behavioural modification happening. As long as responsibleness isn't built among the health-functionaries, it's tough to contain adverse effects and adverse events alongside performance observance and follow-up assessment in place.

The method tailored to execute the training process lack clarity. There's no effort to make associate acceptable training settings that's is tributary to learning, raising considerations, and enhancing the commitment of health- 
functionaries. Thus, in the absence of clear-cut mechanisms for efficient execution, one would be uncertain whether or not new ways, if any, would manufacture higher results.

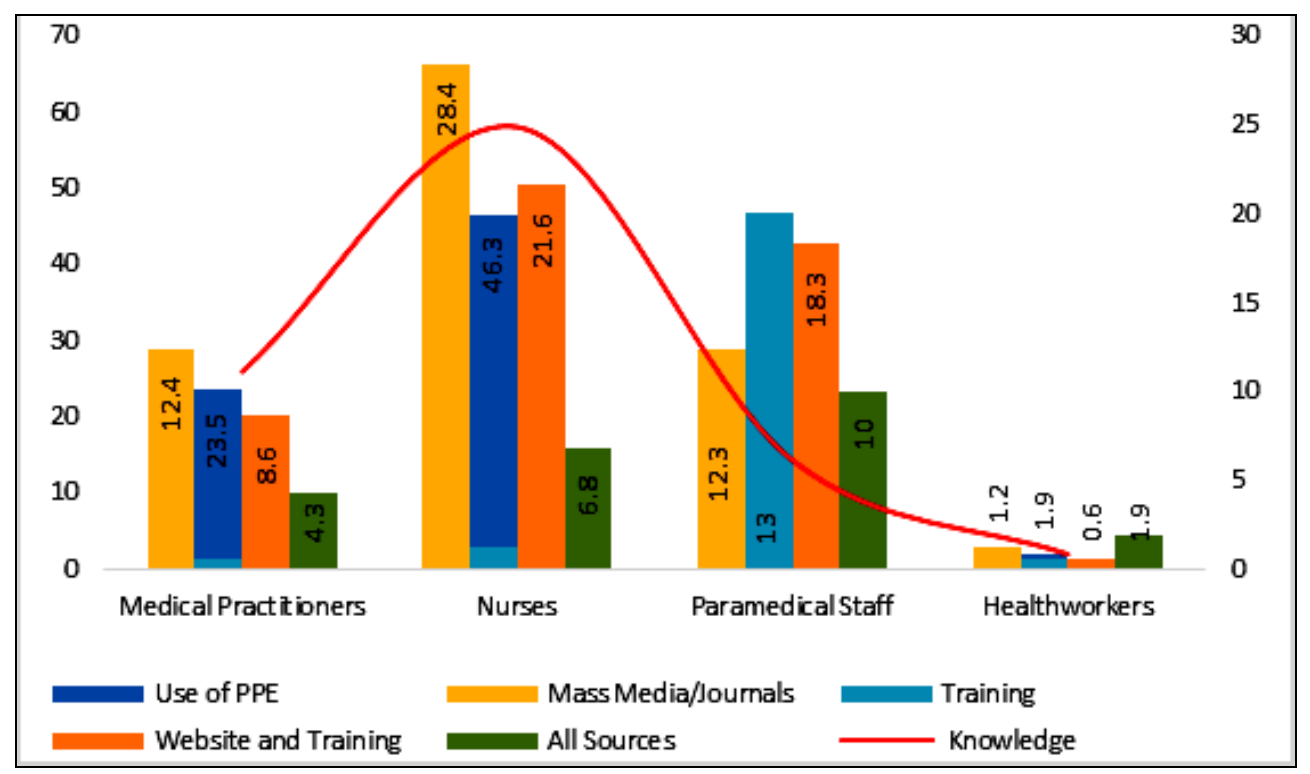

Fig. 1. Knowledge and sources of COVID-19 (in percent)

\section{PERCEIVED KNOWLEDGE, MANAGEMENT AND DELIVERY}

Analysis of Knowledge-Management-Delivery shown that the knowledge of triage in terms of public health importance and connected aspects gazes' 26.8\% to medical-practitioners having confidence in delivering care and treatment whereas, for nurses, it is $60.7 \%$. However, knowledge of triage found deficiency among paramedical staff. Also, the management of symptomatic and asymptomatic to getting less compared to triage. Solely functionaries who have directly associated with patients appear to possess knowledge of managing care. Healthfunctionaries felt that they assured of their skills in screening risk factors, providing treatment, and serving therapeutic care. This has been explained with $68.5 \%$ variability of the dependent variable quantity around its mean. This change once other co-morbidity, $61.3 \%$ of the variation, illustrates case fatality would be high, that does not factor regarding the managing and delivering capabilities. The frequency of positive cases with $60+$ years accounts for $52 \%$ and overall case fatality recorded at $3.3 \%$. The challenges of caring for and managing the aged with co-morbidity, the confidence level of healthfunctionaries appears to be lower. A total of $24.5 \%$ of health-functionaries underwent continuous medical education within the last 2-years. Knowledge-ManagementDelivery analysis related data is shown in Fig 2.

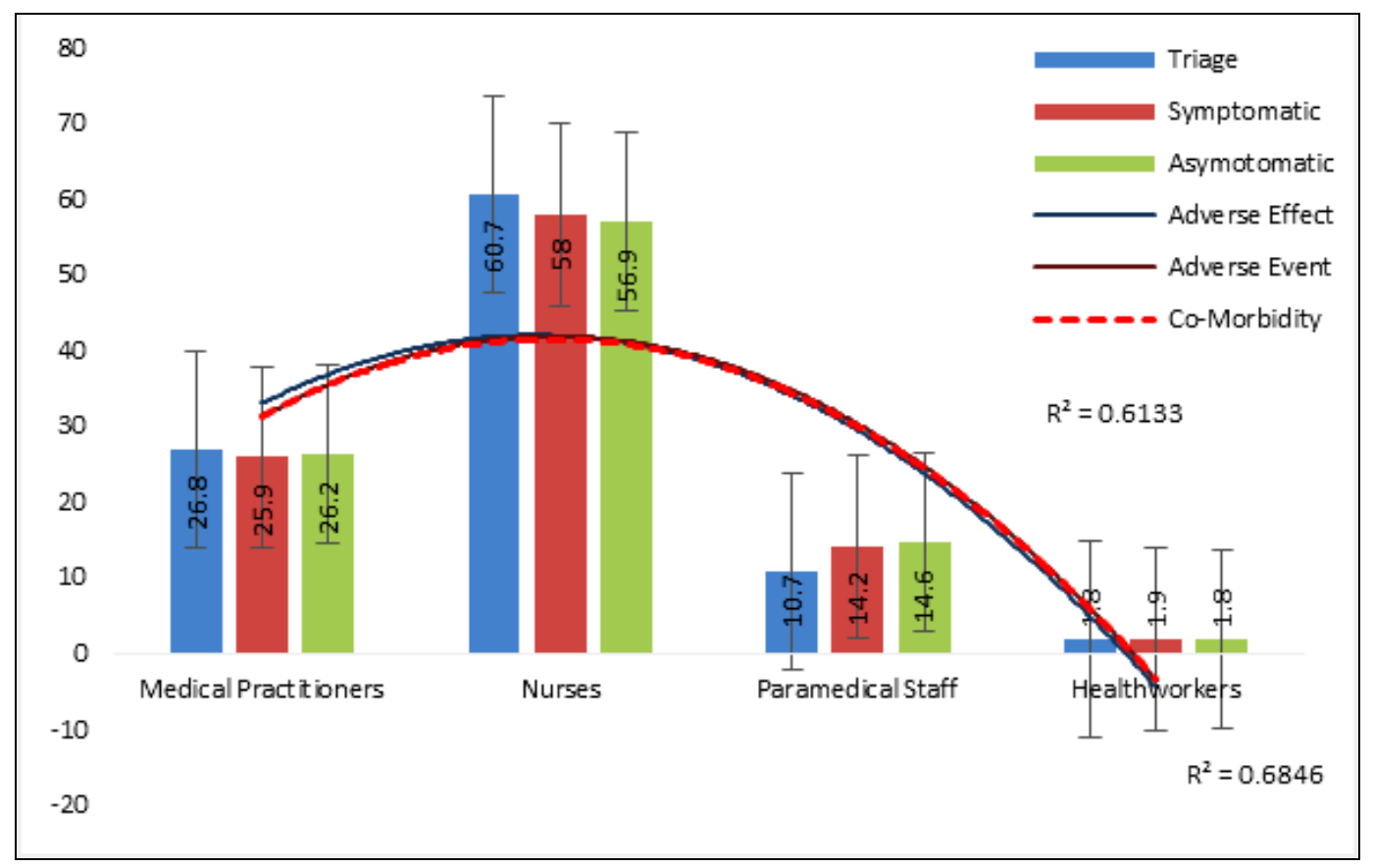

Fig. 2. Perceived knowledge, management and delivery (in percent) 
Given the standard of training, on the one hand, the poor stress on community-based health intervention on the opposite hand, we are ready to not expect healthfunctionaries to perform. Much, as of now, they aren't provided with any in-service training or concern in developing clinical skills such a virulent disease or endemic interventions, which is additionally the cause and questionable. There's a necessity for providing effective training skills across health-functionaries in prevention and management, aside from lockdown and quarantine. The requirement for enhancing competencies and skills was completely perceived by all interviewed.

\section{CONSTRAINTS AND CHALLENGES IN TRAINING}

One-third of the health-functionaries didn't receive any training within the last 5 years. This appears to be
$24.4 \%$ of medical-practitioners and $23.4 \%$ of nurses who are posted in secondary/tertiary setups or a part of the surveillance team. $66.7 \%$ of community health workers, who supposedly First-Contact-Point at the village/ward level didn't attend any training program within the last 5years. This reflects that training receives a very low priority.

Training isn't recognized as an intervention to spice up performance while it's seen in isolation and instructive. None of the health functionaries undergone on training related to infectious diseases like SARS (China), MERS (Middle-East), Ebola (Africa), H1N1-Swine flu (Mexico) within the last five years as these epidemics thought to be a regional basis and can't unfold to the Indian continent. Though $31.6 \%$ undergone communicable disease-related training (Fig. 3), this validates the extent of preparedness for any epidemic.

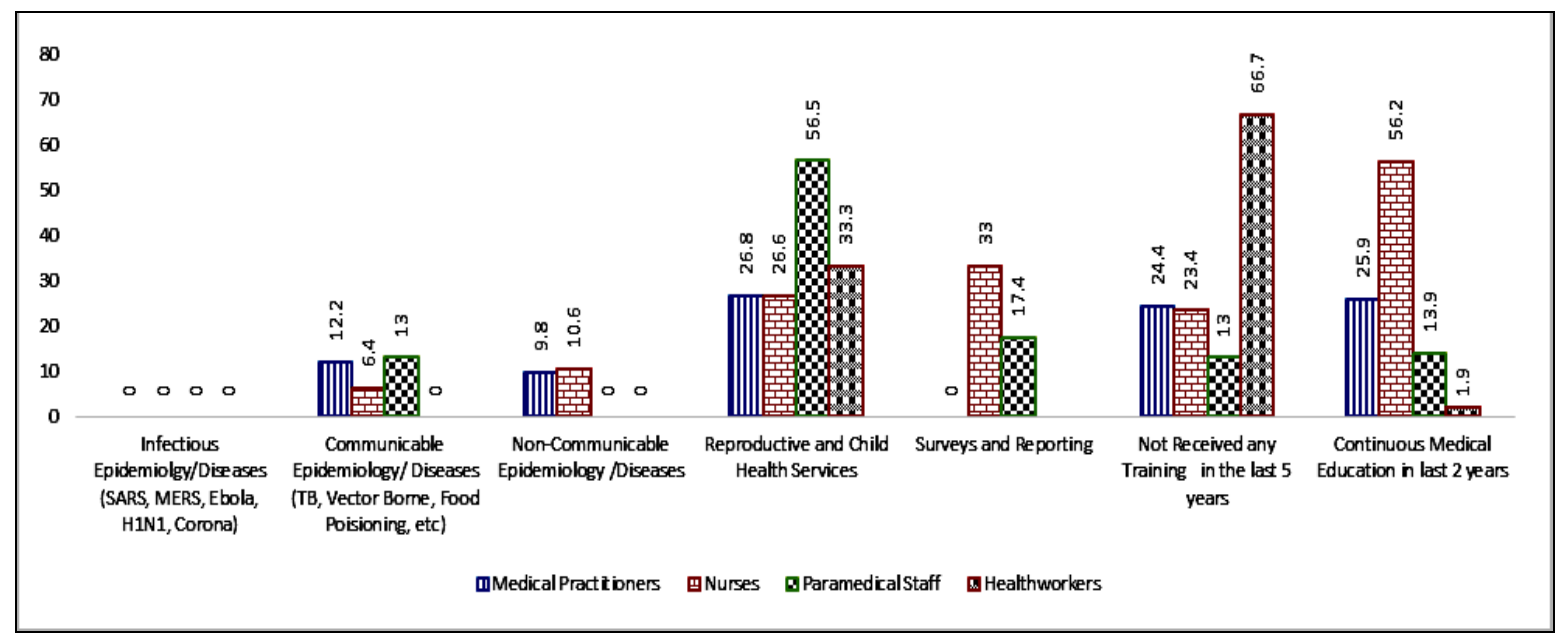

Fig. 3. Status of training and skill orientations (in percent)

The inability to foresee the health problems and incompetent trainers alongside lack of technical steerage resulted in no or less quality orientation caused in lowering the credibility of training. India is excused due to early initiatives to lockdown and tracing the persons-withcoronavirus to quarantine, otherwise, there would be 'corona tsunami' than Americans, Europeans, and Iranians facing, a reality. Excerpts from the interviews suggest training is viewed as constraints in achieving program objectives rather than facilitating them. The need for training is never felt by top management and it's not practiced. Thus, there's a mismatch between the personal and programmatic goals. Over the past 2-decades, the emphasis on quantity, and thus the standard of human resources has taken back seat. This quantity-based approach may accrue the provision of personnel however productivity and performance have remained poor. So, there's a necessity for rethinking and to the repositioning of training, as a lesson given by Coronavirus. The time has come to noticeably draw training as an intervention tool, and important to be positioned for skill development.

\section{SPATIAL AND CAPACITY MAPPING}

Although the availability of physical space and beds; medical equipment, PPE; manpower - caregiving and, pharmacist; capacity - handling several patients; and professionals like medical-practitioners, nurses, and technicians are done through spatial and capacity mapping. The responses seem a very bleak image. $24.7 \%$ of COVID-19 serving medical-practitioners expressed that their hospitals have space, medical equipment, and professional competencies compared to but $17.6 \%$ of an identical cohort of them reported the same. In distinction, $52.6 \%$ of the nurses mentioned that infrastructure is that the downside while $55.2 \%$ of them cited for lack of staff to deal with possible COVID-19 patients. As a result of this, several designated COVID-19 centres carry-out solely diagnostic services and fall-out of any positive-case being referred to tertiary set-ups, reflecting the delay-in diagnostics process and subsequent care and treatment. On the contrary, it's conjointly determined that patients referred to COVID-19 designated centre with none confirmed report of infection despite the very fact that hospitals have the power to treat such patients. This further sinks the availability of the physical and human resources leading to compromise of serious COVID-19 patients. This reflects the extent of training and capability to undertake risk management.

A total of $46.1 \%$ of health-functionaries expressed that they share facilities like washroom, dining, transportation, and corridors with COVID-19 patients, although they've 
got restricted PPE, the worry of getting the infection and conscious of the challenge. A curve-linearity is determined with $1.9 \%$ health workers, $32.1 \%$ of paramedicals, $61.6 \%$ nurses, and $44.5 \%$ the medical-practitioners. Though all health-functionaries follow the safety protocols, still the causality to them is high, as many as one-tenth of a million. There's a discrepancy noted. Not all health functionaries are given PPE and needed facilities being hooked into hierarchy rather than on the extent thereto health-personnel face risk of exposure. This's often a result of nurses and other health workers who don't seem to be a part of the decision-making bodies.

\section{DILUTION OF FAITH VS ANXIETY: THE ROOT OF THE TRAINING?}

Taking cognizance of the various incidents of abuse, attack, stigma \& discrimination on health-functionaries, the study observed that $69.5 \%$ of them experienced such incidents. About $7.4 \%$ of medical-practitioners are abused and attacked while 5.6\% claimed they're stigmatized/ discriminated against and stayed away from their homes. $22.2 \%$ stated they're assaulted and $17.6 \%$ said they're discriminated in their residential community area and asked them to vacate the house sort of a shot.

Attack and abuse don't seem to be new for the healthfunctionaries in a pre-COVID era as a result of relatives thought that patients don't seem to be receiving adequate attention and care. This has forced some hospitals to rent bodyguards for their staff. If the Indian healthcare system would better and more accessible with quality and safety, there has been greater trust in health-functionaries today in an exceedingly pandemic scenario. Within the pre-COVID era, dilution of faith in health-functionaries whereas during the COVID era, anxiety and risk of getting an infection, leading to abuse and discrimination. Federal and State Governments become saviours and amended an Ordinance - Epidemic Diseases Act, 1987 to guard the healthfunctionaries against the perpetrators (Ministry of Law and Justice, 1987). Because of the lack of morals, ethics, and value, there is the commercialization and low-level of care and treatment. This, in turn, stems from training additionally as a system approach to deal with these problems. Candidly, $11.2 \%$ of the functionaries mentioned that their families don't seem to be pleased with the duty within the COVID-19 services. 36.8\% interviewed are unhappy with the awards and security act. Unsympathetic relationship, discriminatory behaviour, and rising frustration levels of the individuals indicates the coronavirus isn't alive, "it is programmed to proliferate, and because it infects more people, it disrupts our lives", said Brahmavihari Swami.

Strengthening the training to reinforce the standard, safety, and effectiveness are at the foremost necessary. Training needs to add how to be calm, positive pro-action; no reaction, nor inaction. The presence of ABCD - anxiety, bothered, concerned, and distressed - lit a fireplace. Swami adds, "while not ignoring the gravity of the coronavirus, uncontrolled reactions, rumours, and fears might make us go haywire". However, something is missing Training, Communication, and Commitment. Because health-functionaries diluted the trust and faith in them, which could be recovered solely by effective communication and efficiency in delivering the services and this might be achieved via quality training. Without training, even we've got the foremost effective technologies however the worst of human beings. Institutions need to be evaluated not just by its technologies, but conjointly by the character of its cadre and thus the training.

\section{CONCLUSION: COVID PRO QUO}

In Sum up, the concentration curve explains that determinants of spatial and capacity-building are line-up for COVID-19 services due to the effect of training sunshine. The concentration curve (Fig. 4) lies above the diagonal as the effect is concentrated among the space, medical instruments, and negative capacity. Whereas, skilled professional and manpower, lies below as training effects appears to be seeding out, as explained by $\mathrm{R}^{2}$ with 65.1 percent. Unlike the range, the concentration curve reflects the experiences of health-functionaries as a whole and not just two extreme groups.

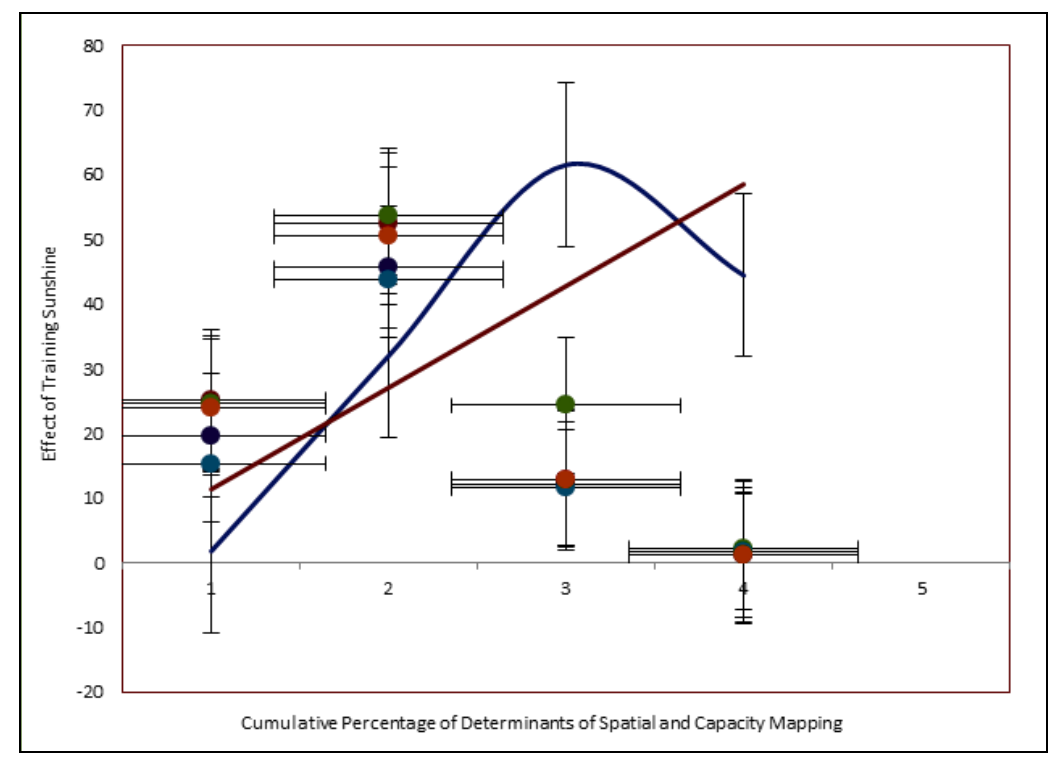

Fig. 4. Measuring training performance - concentration curve 
This explains invisible coronavirus fear something that characterized by a high volume of adverse effects and case fatality rate. Though the recovery seems to be giving hopes, however not having medical miracles to stop the asymptomatic infection, maybe a worry, and a challenge to the competency and skills of the functionaries. It reflects the experiences of the health-functionaries on KMD of COVID-19 services while the concentration curve remembers the extent of decision-making powers of health-functionaries studied. Moreover, the curve closes to the diagonal pronounces that apart from space variables, inequalities in training appear to be non-variant. Neither fear nor the disease has any statistical association with health inequality, suggesting that neither higher spending nor care and treatment would save the individuals falling to the 'health poverty' trap unless specialize in competencies and enhance the skills that occur. This is often due to regressiveness casts a dark shadow over our lives and expressed concern over the progressivity of quality and safety.

It appears that training - competency and skills related inequality and inequity in healthcare exist. On the nature of training, a variety of useful lessons are learned, but a variety of questions remain unanswered when it involves normative training issues. The skill-sets might gradually supersede the necessity for long, laborious, and sometimes focussed formal education. The time is up to differentiate between quantum and quality of learning. True characters - morale, value, and ethics emerge in such crisis, and are mirrored within the selection of competency and therefore the skills. Perhaps coronavirus has thrown up challenges and problems that we couldn't are imagined. However, it's also taught us really important learning of all is our vulnerability; that's we would like to train our self to strengthen our competencies and skills to be self-reliant and self-sufficient.

\section{CONFLICTS OF INTEREST}

The authors declare no conflicts of interest.

\section{DECLARATION}

The contents of this paper are published after receiving a signed copyright agreement from the corresponding author declaring that the contents of this paper are original. In case of any dispute related to the originality of the contents, editors, reviewers and publisher will remain neutral.

\section{REFERENCES}

Ministry of Law and Justice (1987). The Epidemic Diseases (Amendment) Ordinance. The Gazette of India. Government of India. $1987 . \quad$ Online available at https://www.indiacode.nic.in/bitstream/123456789/2326/1/A1 897_03.pdf

Misra S (2020). Integrative Approach Amid COVID -19 Crisis A Perspective. Journal of Vaccines \& Vaccination, 11, 416.

MoHFW (2020). Guidance document on appropriate management of suspect/confirmed cases of COVID-19. Ministry of Health and Family Welfare. Government of India. New Delhi. 2020. Online available at https://www.mohfw.gov.in/pdf/FinalGuidanceonMangaemento fCovidcasesversion2.pdf

\section{How to cite this article?}

Ravichandran N, Shivangi S, Rakesh B (2021). Spatial mapping and enshrined capacity: Assessment of COVID-19 servicing Indian hospitals. Current Medical and Drug Research, 5 (1), Article ID 214. 